\title{
IL-33 modulates inflammatory brain injury but exacerbates systemic immunosuppression following ischemic stroke
}

\author{
Shenpeng R. Zhang, ${ }^{1}$ Marius Piepke, ${ }^{2}$ Hannah X. Chu, ${ }^{1}$ Brad R.S. Broughton, ${ }^{1}$ Raymond Shim, ${ }^{3}$ \\ Connie H.Y. Wong, ${ }^{3}$ Seyoung Lee, ${ }^{1}$ Megan A. Evans, ${ }^{1,4}$ Antony Vinh, ${ }^{1,4}$ Samy Sakkal, ${ }^{5}$ \\ Thiruma V. Arumugam, ${ }^{6,7}$ Tim Magnus, ${ }^{2}$ Samuel Huber, ${ }^{2}$ Mathias Gelderblom, ${ }^{2}$ \\ Grant R. Drummond, ${ }^{1,4}$ Christopher G. Sobey, ${ }^{1,4}$ and Hyun Ah Kim ${ }^{1,4}$ \\ 'Cardiovascular Disease Program, Biomedicine Discovery Institute and Department of Pharmacology, Monash University, \\ Clayton, Victoria, Australia. ${ }^{2}$ University Medical Center Hamburg-Eppendorf, Hamburg, Germany. ${ }^{3}$ Centre for Inflammatory \\ Diseases, Department of Medicine, Monash University, Monash Medical Centre, Clayton, Victoria, Australia. ${ }^{4}$ Department \\ of Physiology, Anatomy \& Microbiology, School of Life Sciences, La Trobe University, Bundoora, Victoria, Australia. ${ }^{5}$ College \\ of Health and Biomedicine, Victoria University, Western Centre for Health, Research and Education, St. Albans, Victoria, \\ Australia. ${ }^{6}$ Department of Physiology, Yong Loo Lin School of Medicine, National University of Singapore, Singapore. \\ ${ }^{7}$ School of Pharmacy, Sungkyunkwan University, Suwon, South Korea.
}

Stroke triggers a complex inflammatory process in which the balance between pro- and antiinflammatory mediators is critical for the development of the brain infarct. However, systemic changes may also occur in parallel with brain inflammation. Here we demonstrate that administration of recombinant IL-33, a recently described member of the IL-1 superfamily of cytokines, promotes Th2-type effects following focal ischemic stroke, resulting in increased plasma levels of Th2-type cytokines and fewer proinflammatory (3-nitrotyrosine ${ }^{+} \mathrm{F} 4 / 80^{+}$) microglia/macrophages in the brain. These effects of IL-33 were associated with reduced infarct size, fewer activated microglia and infiltrating cytotoxic (natural killer-like) T cells, and more IL-10-expressing regulatory T cells. Despite these neuroprotective effects, mice treated with IL-33 displayed exacerbated post-stroke lung bacterial infection in association with greater functional deficits and mortality at $\mathbf{2 4}$ hours. Supplementary antibiotics (gentamicin and ampicillin) mitigated these systemic effects of IL-33 after stroke. Our findings highlight the complex nature of the inflammatory mechanisms differentially activated in the brain and periphery during the acute phase after ischemic stroke. The data indicate that a Th2-promoting agent can provide neuroprotection without adverse systemic effects when given in combination with antibiotics.

Authorship note: CGS and HAK contributed equally to this work.

Conflict of interest: The authors have declared that no conflict of interest exists.

Submitted: April 9, 2018 Accepted: August 7, 2018 Published: September 20, 2018

Reference information: JCI Insight. 2018;3(18):e121560. https://doi.org/10.1172/jici. insight.121560.

\section{Introduction}

Ischemic stroke triggers an extensive inflammatory response in the brain that is thought to be a major mechanism of secondary brain injury. This occurs when cerebral ischemia induces the activation and proliferation of brain-resident microglia and the recruitment of circulating leukocytes, including neutrophils, monocytes, and lymphocytes $(1,2)$. This acute local inflammatory response is mediated by cytokines and chemokines initially released by ischemic cerebral endothelial cells (3). High levels of proinflammatory cytokines and/or low levels of antiinflammatory cytokines are linked with early worsening and more severe outcomes of stroke $(4,5)$.

Clinical evidence indicates that elevated numbers of circulating Th2-related cells are associated with a reduced risk of cardiovascular events (6). Unlike Th1 responses, which promote the generation of proinflammatory mediators in the setting of cardiovascular disease, Th2-type responses lead to production of antiinflammatory cytokines, such as IL-4, IL-5, IL-9, IL-13, IL-25, and IL-33 (7, 8). Importantly, the relative predominance of a Th1- versus a Th2-type inflammatory response appears to be crucial for the composition and activity of immune cells entering the brain and consequently for the severity of the 
eventual outcome after cerebral ischemia (9). Mice lacking IL-4 develop more M1-polarized microglia/ macrophages, larger infarcts, and worse functional deficits following cerebral ischemia, whereas administration of recombinant IL-4 abrogates these effects (10) and improves outcome in WT mice $(10,11)$. Consistent with such an effect, IL-10, which can mediate Th2 cell functions (7), also exerts protective effects whereby its overexpression results in smaller infarcts $(12,13)$. Conversely, deficiency of IL-10 results in more severe infarcts and functional deficits after stroke (14).

Here, we have tested the hypothesis that pharmacological intervention to modulate the profile of inflammatory mediators using a Th2-promoting cytokine, recombinant IL-33, can limit brain injury and functional impairment during the early phases after stroke. IL-33 is a ligand for suppression of tumorigenicity 2 (ST2), an IL-1 family receptor, and can initiate and amplify Th2-type responses (7, 15). A transmembrane form of ST2 is expressed primarily on Th2 cells to mediate its Th2 effector functions (16). IL-33 can also activate other cell types besides T cells, including mast cells, macrophages, dendritic cells, eosinophils, basophils, NKT cells, and innate-like lymphoid cells, that could contribute to amplification of a Th2-type response (17). In pathological settings, endogenous IL-33 can be released from necrotic cells and degraded by caspases after apoptosis (18).

Protective effects of IL-33 administration have been reported in experimental disease models such as myocardial infarction (19), atherosclerosis (20), inflammatory bowel disease (21), Alzheimer's disease (22), experimental autoimmune encephalomyelitis (23), and concanavalin A-induced liver damage (24). Further, in tumor-bearing mice, IL-33 suppresses activity of NK cells and dendritic cell maturation, and enhances M2 polarization of macrophages (24). Here, in a mouse model of cerebral ischemia, we have extensively investigated the therapeutic potential of recombinant IL-33 (and in limited studies, IL-4) to enhance Th2-associated inflammatory signaling and thus limit brain injury and clinical impairment. Daily administration of IL-33 $(2 \mu \mathrm{g} / \mathrm{d})$ and IL-4 $(5 \mu \mathrm{g} / \mathrm{d})$ have each been used for therapy in non-stroke experimental studies $(25,26)$, and so here they were first administered at these doses over 2 days. While these interventions were found to be neuroprotective in association with Th2-related antiinflammatory actions, they also accelerated and/or exacerbated post-stroke Th2-mediated systemic immunosuppression (27-29), leading to lung infection and mortality. These adverse systemic effects were mitigated either by combination therapy with antibiotics or by reducing the dose of IL-33 by $80 \%(0.4 \mu \mathrm{g} / \mathrm{d}$ for $2 \mathrm{days})$ and the ischemic period from 60 to 45 minutes. This latter modification also enabled 72-hour follow-up and an examination of the role of IL-10 generation and/or Tregs.

\section{Results}

Effects of IL-4 or IL-33 on stroke outcome. Blood flow to the cortical region supplied by the middle cerebral artery (MCA) was reduced by $\sim 80 \%$ during MCA occlusion (MCAO), and reperfusion resulted in the prompt return of flow to pre-ischemic levels (Supplemental Figure 1A; supplemental material available online with this article; https://doi.org/10.1172/jci.insight.121560DS1). Compared with vehicle, neither IL-4 nor IL-33 had any effect on blood flow changes (Supplemental Figure 1A). Representative coronal brain sections at 24 hours after MCAO are shown in Figure 1, A-C. Mice treated with IL-4 (5 g daily; total dose, $10 \mu \mathrm{g}$ ) or IL-33 ( $2 \mu \mathrm{g}$ daily; total dose, $4 \mu \mathrm{g}$ ) had $\sim 35 \%$ smaller infarcts than vehicle-treated mice (Figure 1D), due mainly to smaller cortical infarcts (Figure 1, A-C, and Supplemental Figure 1, D and E).

Vehicle-treated mice subjected to stroke displayed clinical deficits, and reduced hanging grip time and locomotor activity compared with sham-operated mice (Figure 1, E-G). Treatment with IL-33 or IL-4 augmented the clinical deficits and tended to further reduce hanging grip time and locomotor activity after stroke compared with vehicle treatment (Figure 1, E-G, and Supplemental Figure 1, B and C).

Effect of IL-33 on Th1 and Th2 cytokines. The Th1-associated (as opposed to Th2-associated) nature of acute postischemic brain inflammation in C57BL/6 mice was demonstrated by marked increases in expression of the proinflammatory cytokines $\mathrm{Il}-1$ and $\mathrm{Il}-6$ (Figure 2, A and B). By contrast, brain expression of the antiinflammatory Th2 cytokines $\mathrm{Il}-5$ and $\mathrm{Il}-13$ were unaffected by stroke (Figure 2, C and D). Further, administration of IL-33 was confirmed to exert a Th2-promoting effect in vivo by increasing brain expression of the antiinflammatory Th2 cytokines $\mathrm{Il}-5$ in stroke-operated and $\mathrm{Il}-13$ in sham-operated mice (Figure 2, C and D), while profoundly reducing post-stroke expression of $I l-1 \beta$ and $I l-6$ (Figure 2, A and B). Stroke tended to increase the systemic expression of G-CSF, TNF, and IL-6, but plasma levels of these cytokines were unaffected by IL-33 (Figure 2, E-G). By contrast, treatment with IL-33 increased plasma levels of IL-5 protein by $\sim 400$-fold in sham and stroke mice (Figure $2 \mathrm{H}$ ). 


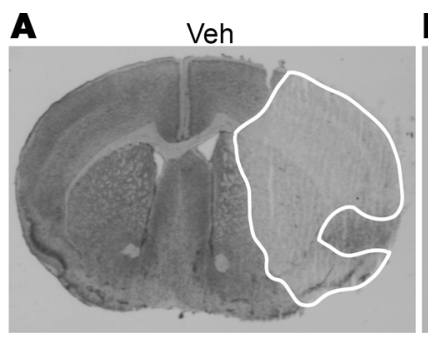

D

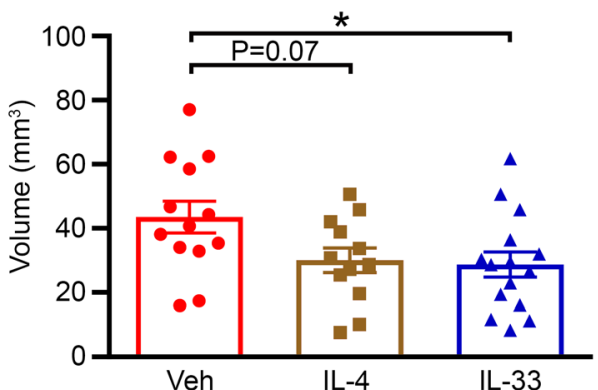

$\mathbf{F}$

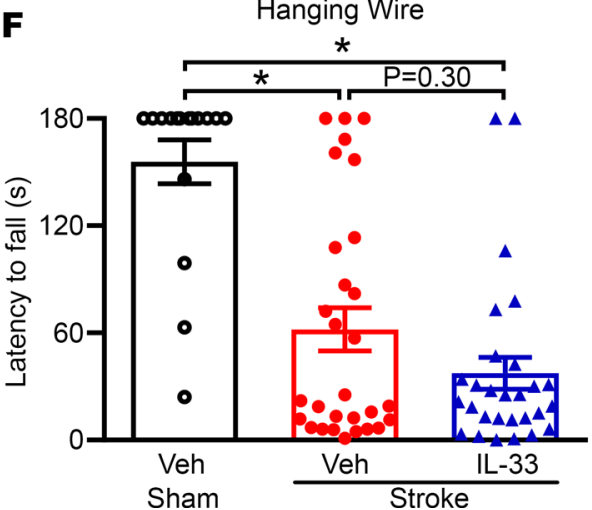

B

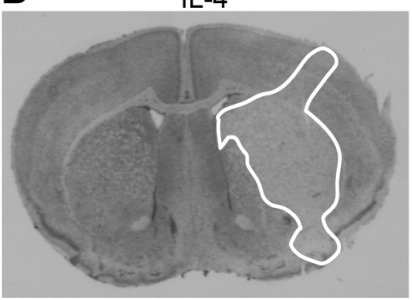

$\mathbf{E}$
C
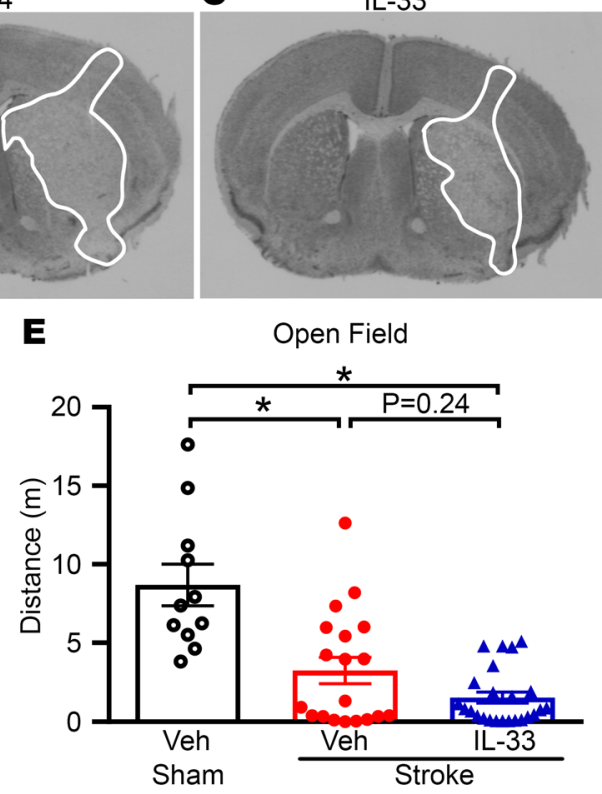

G

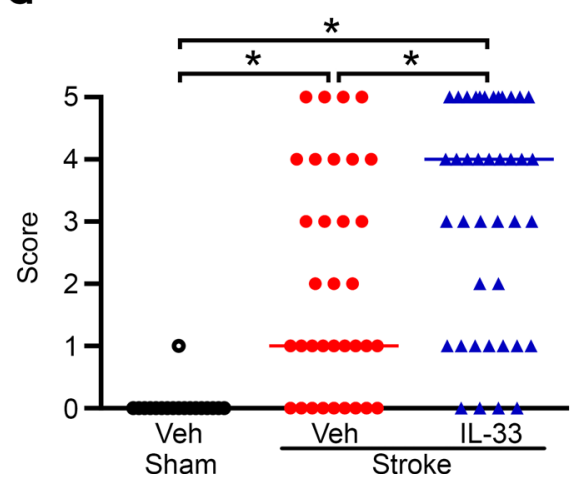

Figure 1. Infarct volume and functional outcome. Representative coronal brain sections from mice treated with (A) vehicle (Veh), (B) recombinant IL-4, or (C) recombinant IL-33 after stroke (infarct areas are outlined in white). Total infarct volume (D), open field performance $(\mathbf{E})$, hanging wire latency to fall $(\mathbf{F})$, and clinical score at 24 hours after stroke (C). Data are presented as mean \pm SEM in $\mathbf{D}-\mathbf{F}$ and as median scores in G. ${ }^{*} P<0.05$, 1-way ANOVA with Bonferroni's post hoc test for $\mathbf{D}-\mathbf{F}$ and Kruskal-Wallis test with Dunn's post-hoc test for $\mathbf{G}$. Infarct volume, $n=12-15$; open field, $n=$ 11-24; hanging wire, $n=12-29$; and clinical score, $n=18-40$.

Effect of IL-33 on brain infiltration of immune cells after cerebral ischemia. At 24 hours, there were 7-fold more leukocytes present in the ischemic hemisphere of vehicle-treated mice than in sham controls (Figure 3A). IL-33 treatment had no overall effect on the total number of leukocytes present in the brain at 24 hours (Figure 3A). However, among the $\sim 15,000$ leukocytes $\left(C D 45^{\text {hi }}\right), \sim 20 \%$ were neutrophils, and treatment with IL-33 tended to further increase this number (Figure 3B). Total monocytes present in the brain were increased by $\sim 10$-fold after stroke in vehicle-treated mice, and this increase was attenuated by IL-33 treatment (Figure 3C and Supplemental Figure 2A). While IL-33 had no effect on the stroke-induced increase in $\mathrm{CD}^{+} \mathrm{T}$ cells (Figure 3D), the treatment substantially reduced the numbers of $\mathrm{CD}^{+} \mathrm{T}$ cells, $\mathrm{CD}^{-} \mathrm{CD}^{-}$ double-negative (DN) T cells, NK cells, and NKT cells infiltrating the brain after stroke (Figure 3, E-H, and Supplemental Figure 2, B and C). The effect of IL-33 on NKT cells (Figure 3H) was largely confined to the $\mathrm{CD}^{+}$NKT cell population (Figure 3, I and J, and Supplemental Figure 2D). CXCL16 is a chemoattractant for activated $\mathrm{CD}^{+} \mathrm{T}$ cells and NKT cells, and indeed immunohistochemical staining revealed that this effect of IL-33 was associated with fewer CXCL16 ${ }^{+}$cells present after stroke (Figure $3 \mathrm{~K}$ ).

Effect of IL-33 on microglial/macrophage activation and polarization after cerebral ischemia. Flow cytometry indicated a trend toward a $50 \%$ increase in microglia $\left(\mathrm{CD} 45^{\mathrm{med}} \mathrm{CD} 11 \mathrm{~b}^{+} \mathrm{F} 4 / 80^{+} ; P>0.05\right)$ present at 24 hours 

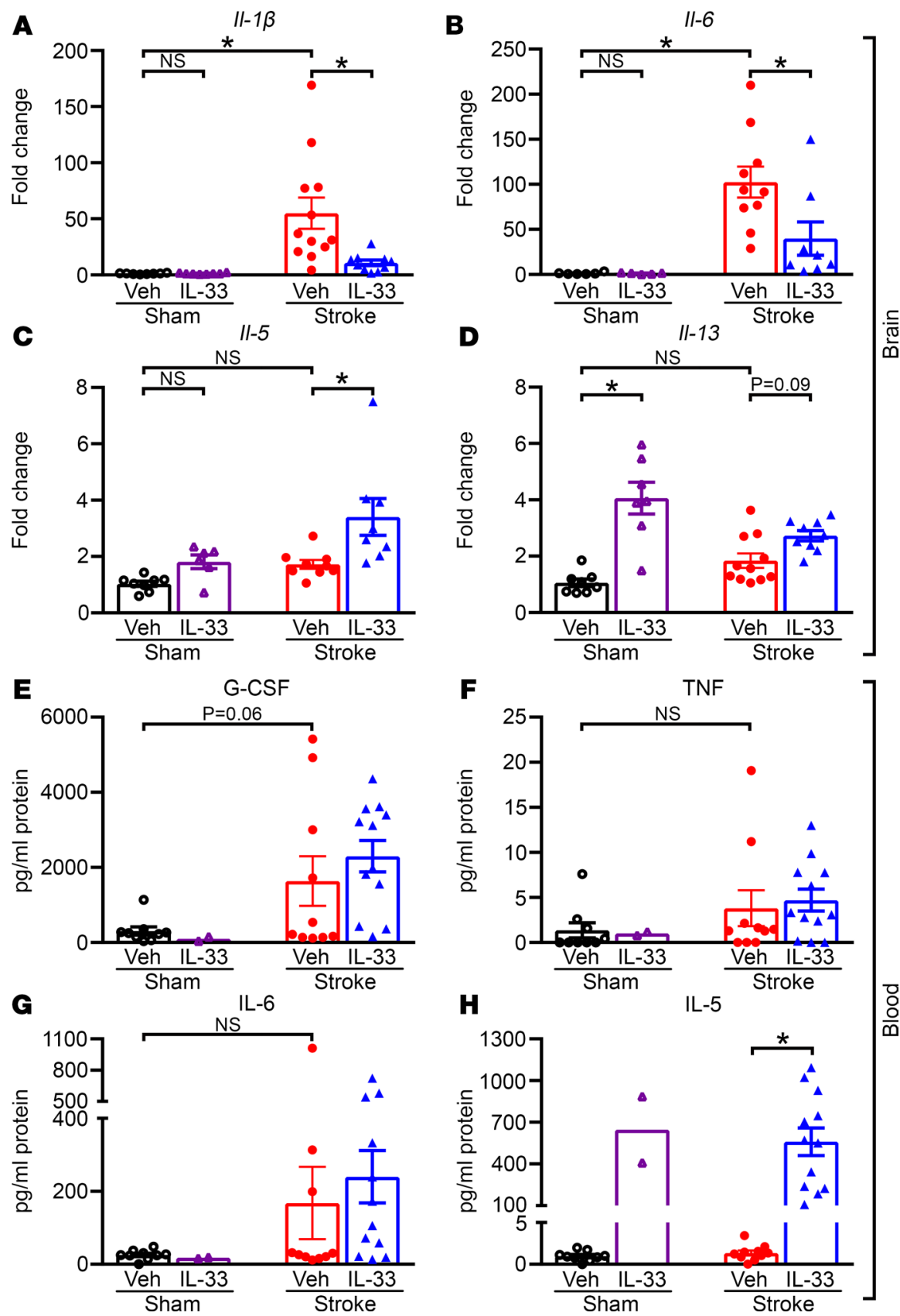

Figure 2. Pro- and antiinflammatory cytokine expression in the brain and plasma after stroke. Expression of (A) II-1 $\beta$, (B) II-6, (C) II-5, and (D) II-13, in the ischemic hemisphere at 24 hours after stroke, quantified using qPCR. Circulating levels of (E) G-CSF, (F) TNF, (G) IL-6, and (H) IL-5 at 24 hours after stroke. Data are presented as mean \pm SEM. ${ }^{*} P<0.05$, 1-way ANOVA with Bonferroni post hoc test. Brain qPCR, $n=5-12$; plasma cytokine: $n=2-12(n=2$ for sham IL-33 only). Veh, Vehicle.

in the ischemic hemisphere of vehicle-treated mice, whereas no such trend was present in IL-33-treated mice after stroke (Figure 4A). Moreover, immunohistochemistry revealed that stroke increased the number of activated microglia/macrophages and that this increase was partly attenuated by IL-33 treatment (Figure 4B, $P=0.07$; Supplemental Figure 3A; $P=0.07$ ). Both flow cytometry (Figure $4 \mathrm{C}$ ) and immunohistochemistry (Figure $4 \mathrm{D}$ ) indicated that there was a $\sim 5$-fold increase in macrophages in the brain following stroke. Furthermore, IL-33 tended to reduce macrophage content (Figure 4, C and D), and there were $\sim 50 \%$ fewer proinflammatory (3-nitrotyrosine ${ }^{+}\left[3-\mathrm{NT}^{+}\right] \mathrm{F} 4 / 80^{+}$) microglia/macrophages present in the ischemic brains of mice treated with IL-33 versus vehicle (Figure 4E and Supplemental Figure 3B). 
A
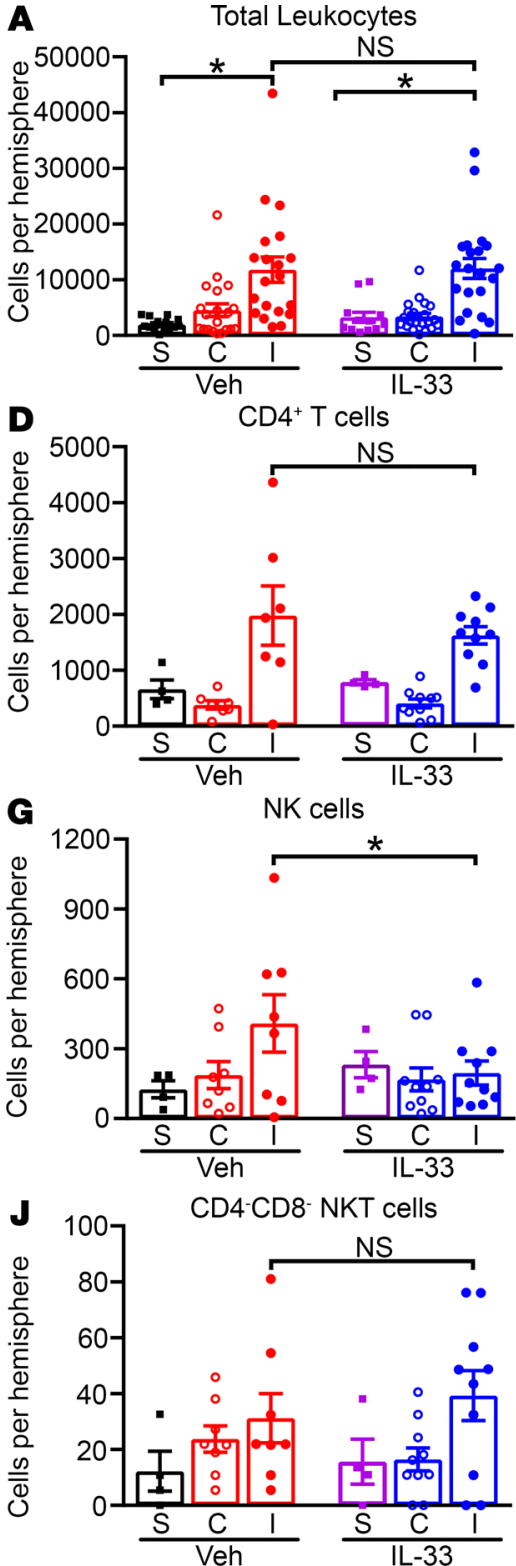

B

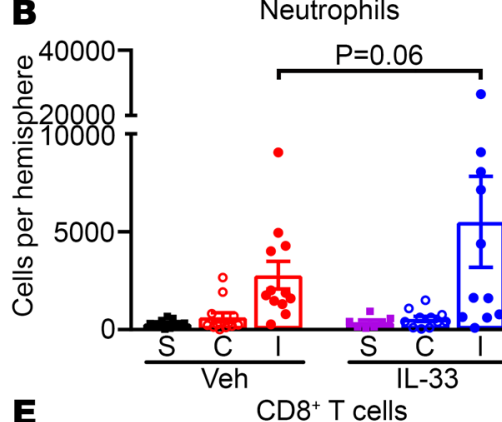

$\mathbf{E}$

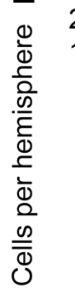
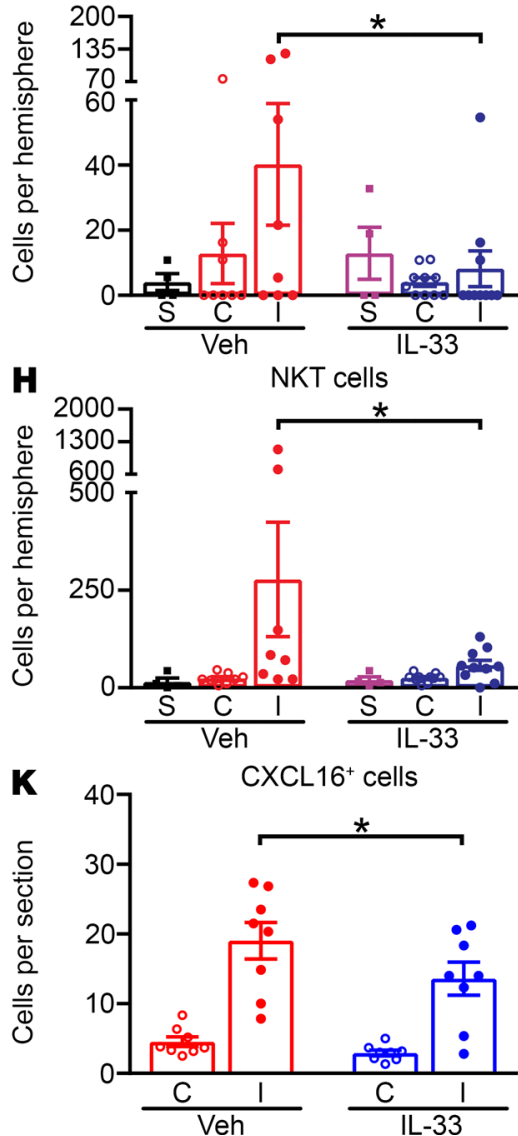
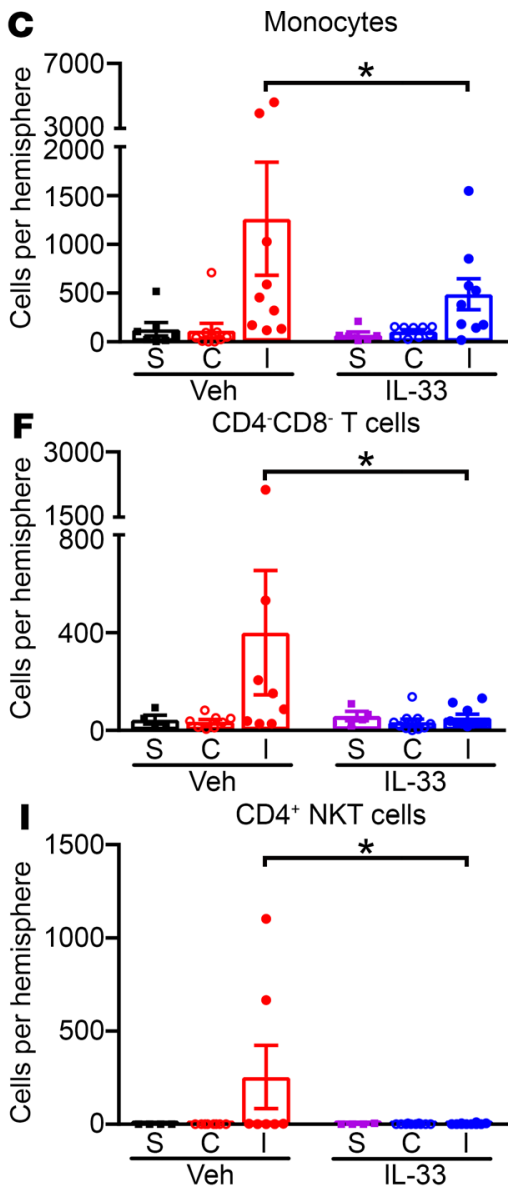

Figure 3. Leukocytes in the brain after stroke. Flow cytometric quantification of (A) total leukocytes, (B) neutrophils, (C) monocytes, (D) CD4+ $T$ cells, (E) CD8+ T cells, (F) CD4-CD8- double-negative T cells, (G) NK cells, (H) NKT cells, (I) CD4+ NKT cells, and (J) CD4-CD8- double-negative NKT cells in each hemisphere at 24 hours after stroke. (K) Immunohistochemical staining of CXCL16+ cells in the brain at 24 hours after stroke. Data are presented as mean \pm SEM. ${ }^{*} P<0.05$, 1-way ANOVA with Bonferroni's post hoc test. Total leukocytes, $n=12-21 ;$ subsets, $n=8-13 ;$ CXCL16 immunohistochemistry, $n=8$. S, sham; C, contralateral; I, ischemic; Veh, vehicle.

Effect of IL-33 on splenocytes and circulating immune cells after cerebral ischemia. Treatment with IL-33 increased spleen weight by $\sim 25 \%$ compared with vehicle controls in both sham- and stroke-operated mice (Supplemental Figure 4A, both $P<0.05$ ), as has been reported (15). Spleen weight was reduced by $10 \%-$ $15 \%$ at 24 hours after stroke in mice treated with either vehicle or IL-33, and this reduction was statistically significant in the latter treatment group (Supplemental Figure 4A). At 24 hours after stroke in both vehicle- and IL-33-treated mice, there were $\sim 40 \%$ fewer splenocytes than in sham-operated mice, and this reduction was statistically significant with IL-33 treatment (Supplemental Figure 4B). This trend toward a reduction in total splenocytes at 24 hours after stroke was notable among CD4 ${ }^{+} \mathrm{T}$ cell and NK cell subsets (Supplemental Figure 4, C and D), but not in NKT cells, macrophages, or monocytes (Supplemental Figure 4, E-G). Treatment with IL-33 generally had no effect on numbers of any splenocytes examined, except for a 2- to 4-fold increase in macrophages and monocytes after stroke (Supplemental Figure 4, B-G). 

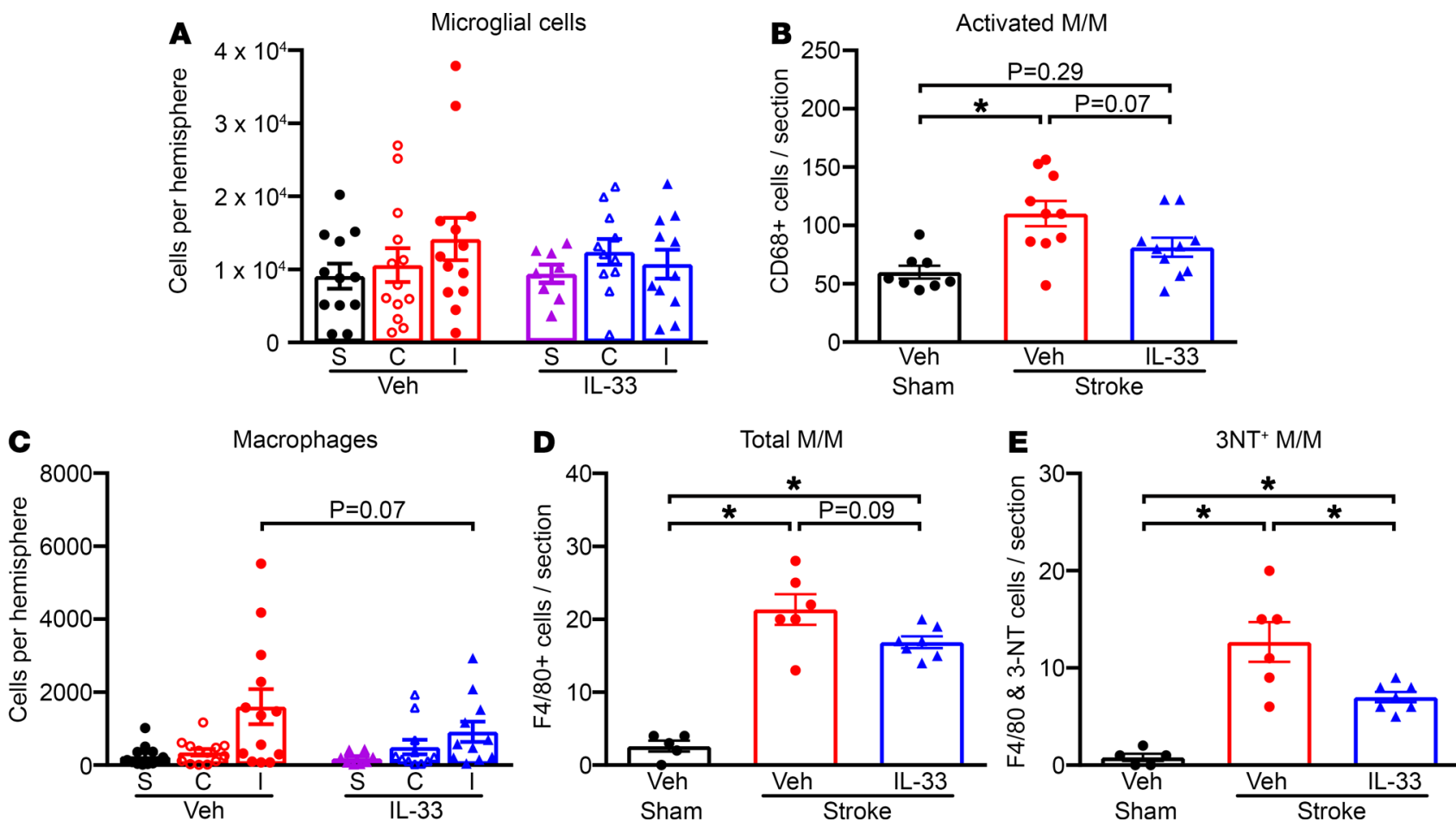

Figure 4. Microglial cells and macrophages in the brain after stroke. Total number of microglial cells quantified using (A) flow cytometry and (B) immunohistochemical staining of $\mathrm{CD}^{+} 8^{+}$cells in the ischemic hemisphere at 24 hours after stroke. Total number of macrophages and/or microglia quantified using (C) flow cytometry and (D) immunohistochemical staining of F4/80+ cells in the ischemic hemisphere at 24 hours after stroke. (E) The number of $\mathrm{F} 4 / 80^{+}$cells that coexpress 3-nitrotyrosine (3-NT) in the ischemic hemisphere is also shown. Data are presented as mean \pm SEM. ${ }^{*} P<0.05,1$-way ANOVA with Bonferroni's post hoc test. Flow cytometry, $n=8-13$; CD68, $n=8-10$; F4/80 and 3-NT, $n=5-7$. S, sham; C, contralateral; I, ischemic; M/M, microglia/macrophages.

Consistent with the peripheral effects of stroke to reduce splenocyte numbers, the number of circulating leukocytes also tended to be reduced (by $30 \%-40 \%$ ) at 24 hours in both vehicle- and IL-33-treated mice, compared with sham mice (Supplemental Figure 5A). There were no statistically significant effects of stroke and/or IL-33 detected among the different cell populations studied, although circulating $\mathrm{CD} 4^{+} \mathrm{T}$ cells were typically $\sim 40 \%$ fewer after stroke in each group (Supplemental Figure $5, \mathrm{~B}-\mathrm{F}$ ).

Effect of IL-33 on post-stroke mortality, functional deficits, and lung infection. Despite the clear effects of the Th2-promoting IL-33 treatment to reduce infarct size in association with reduced Th1-type inflammation in the brain and periphery among survivors (Figure 1, A-D), we were surprised that IL-33 exacerbated clinical score and reduced hanging grip time in comparison to vehicle treatment (Figure 1, E-G). This exacerbation of post-stroke morbidity in mice administered IL-33 was accompanied by a tendency toward a greater post-stroke mortality rate at 24 hours (Figure $5 \mathrm{~A}$ ).

Post-stroke immunodepression and infection is an emerging concept in the understanding of stroke outcomes, and it is associated with a shift in the environment of the immune system from a Th1-driven (proinflammatory) one in the initial stages toward a Th2-skewed (antiinflammatory) setting during the subacute period after stroke (30). We thus investigated the effect of stroke on lung infection at 24 hours in our model and the potential impact of IL-33 treatment. There was virtually no evidence of bacterial infection in lung homogenates from any sham-operated mice treated with either vehicle or IL-33 (Figure 5B). However, consistent with the known consequences of post-stroke immunodepression (31), lung infection was commonly detected in vehicle-treated mice after stroke (Figure 5B). Furthermore, the bacterial load in the lungs was increased by a further 5 -fold in mice subjected to stroke and treated with IL-33 (Figure 5B). We then demonstrated that post-stroke lung infection could be prevented if IL-33treated mice were supplemented with antibiotics (ampicillin and gentamicin; Figure 5B). This combination therapy also reduced mortality and clinical and functional deficits to levels similar to (but not less than) those observed in vehicle-treated mice subjected to stroke (Figure 5, A, C, and D). Importantly, we confirmed that IL-33 therapy in combination with antibiotics reduced infarct size when assessed at 
A

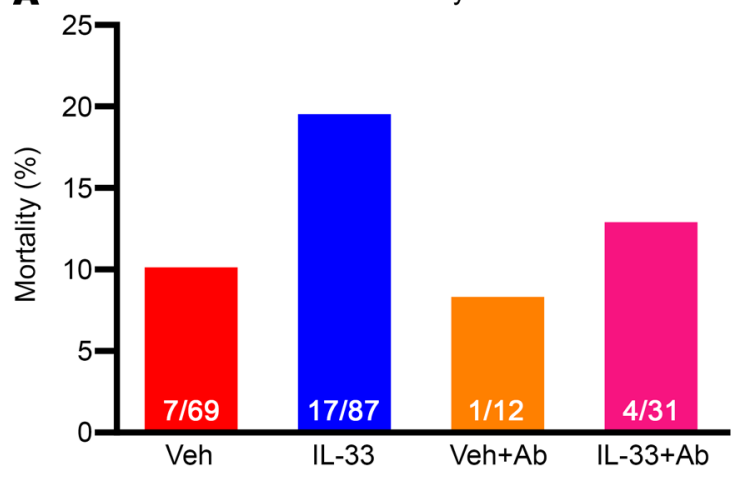

C

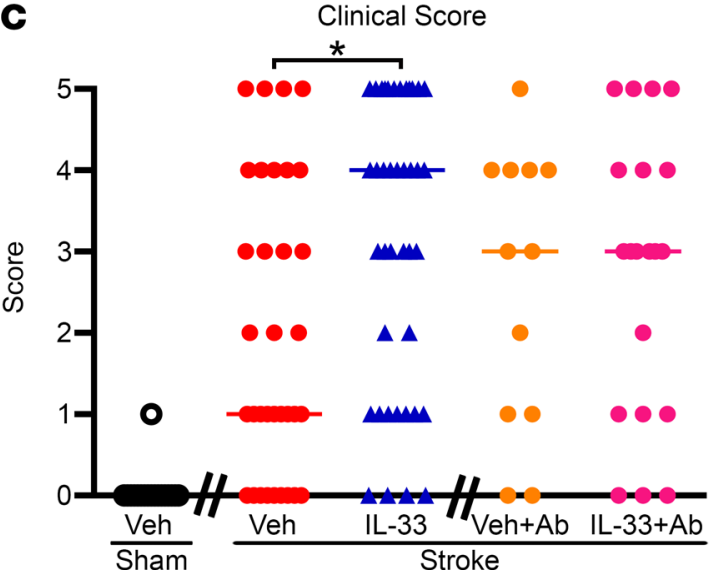

$\mathbf{B}$

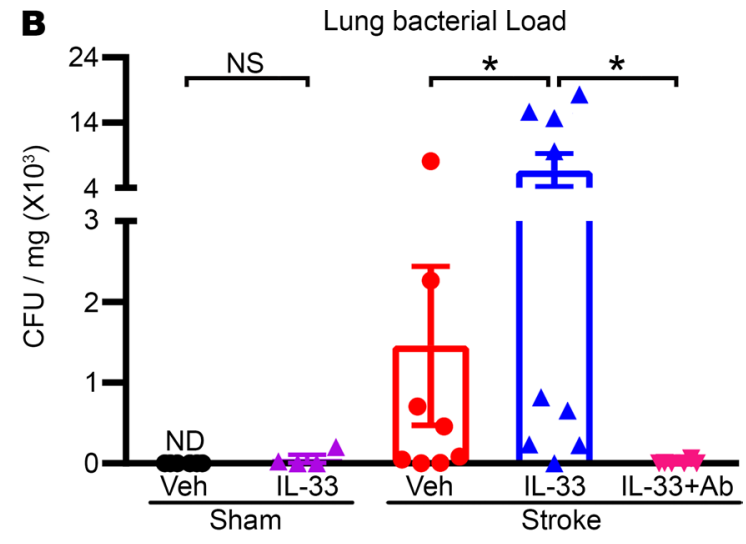

D

Hanging Wire

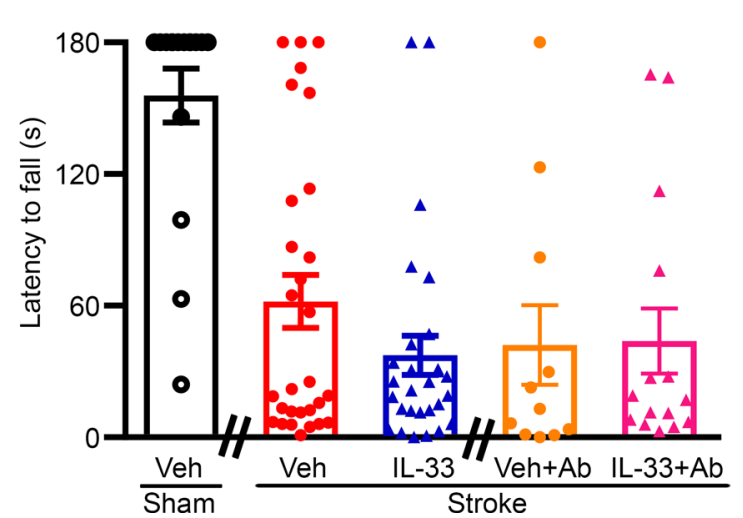

Figure 5. Post-stroke infection and combined treatment with antibiotics. (A) Mortality, (B) bacterial load in the lung at 24 hours after stroke, (C) clinical score, and (D) hanging wire latency to fall at 24 hours after stroke. Data are presented as mean \pm SEM in $\mathbf{B}$ and $\mathbf{D}$ and as median score in $\mathbf{C}$. ${ }^{*} P<0.05$, 1-way ANOVA with Bonferroni's post hoc test for $\mathbf{B}$ and $\mathbf{D}$, and Kruskal-Wallis test with Dunn's post hoc test for $\mathbf{C}$. Bacterial load, $n=4-9 ;$ clinical score, $n=12-40$; hanging wire, $n=11-25$. Ab, antibiotics; ND, not detectable; Veh, vehicle; HW, hanging wire.

either 24 or 72 hours after stroke and when treatment was initiated after cerebral ischemia (2 $\mu \mathrm{g}$ IL-33 daily; total dose, $6 \mu \mathrm{g}$ in 72-hour cohort; Figure 6, A-F).

We similarly demonstrated in T-bet ${ }^{-1-}$ mice, which lack Th1-type cytokines, that an enhanced Th2 immune response caused by IL-33 following stroke (in the absence of Th1 immunity) is detrimental, and resulted in $>70 \%$ (13 of 18) mortality (see also Supplemental Figure 6A). Consistent with our data in C57BL/6 mice (Figure 1, A-E), IL-33 administration resulted in neuroprotection of T-bet ${ }^{-1}$ survivors despite the worsened clinical deficit (Supplemental Figure 6, A and B).

Effect of IL-33 on IL-10 expression by infiltrating immune cells. Despite its overall neuroprotective effects (e.g., Figure 1, A-D), the exacerbation of post-stroke morbidity and mortality by IL-33 without antibiotics (Figure 1, E-G, and Figure 5, A-D) limited our ability to explore the underlying mechanisms of neuroprotection beyond the acute phase of stroke. Therefore, in subsequent experiments we administered a 5-fold-lower daily dose of IL-33 ( $0.4 \mu \mathrm{g})$ to separate cohorts of mice, and examined potential target populations of cells infiltrating the ischemic brain over 3 days and responding to IL-33. For these studies, we utilized IL-10/Foxp-3 double reporter mice in order to identify cells expressing the antiinflammatory cytokine IL-10 and/or the Treg marker FoxP3. We also induced a milder level of cerebral ischemia by using 45 minutes rather than 60 minutes of MCAO. Thus, in these experiments, there was no mortality over 3 days in IL-33-treated mice (vs. 2 deaths in vehicle-treated mice; Figure 7A) and no effect of IL-33 on clinical deficit (Figure 7B). In a separate cohort of mice, we confirmed that this lower dose of IL-33 did not exacerbate the systemic effects of stroke (i.e., lung infection, latency to fall in hanging wire test, clinical score) while tending to exert a level of neuroprotection similar to that of the higher dose (Supplemental Figure 7).

We considered Tregs to be a candidate cell type for mediating neuroprotective effects of IL-33 after stroke. Indeed, IL-33 treatment resulted in a $>3$-fold increase in the number of infiltrating Tregs in the brain 
A

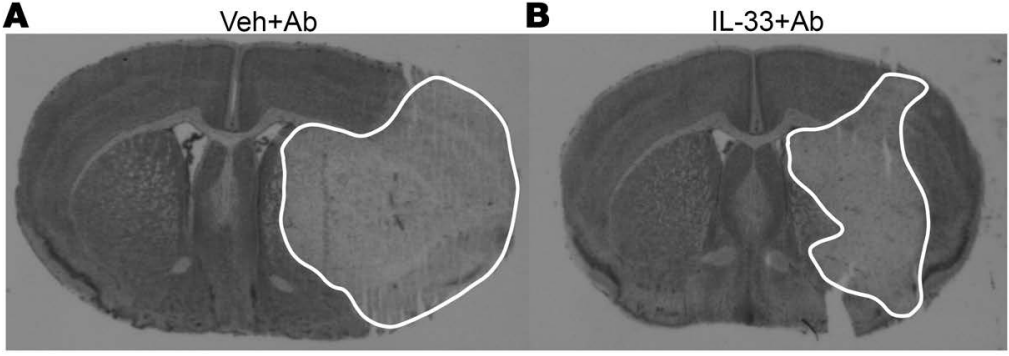

D

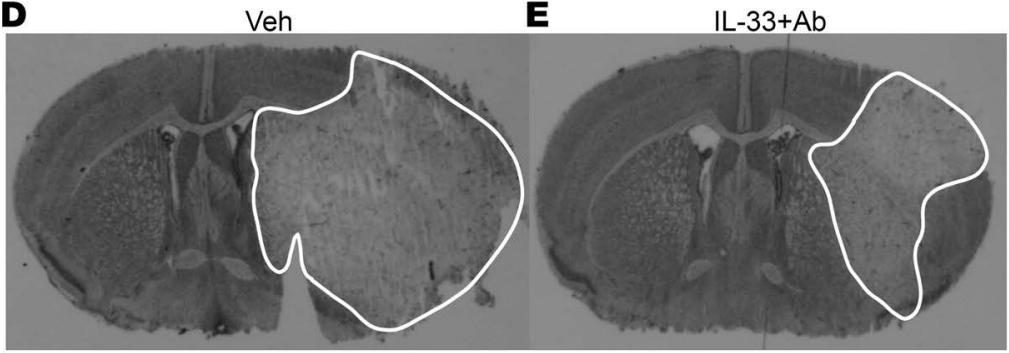

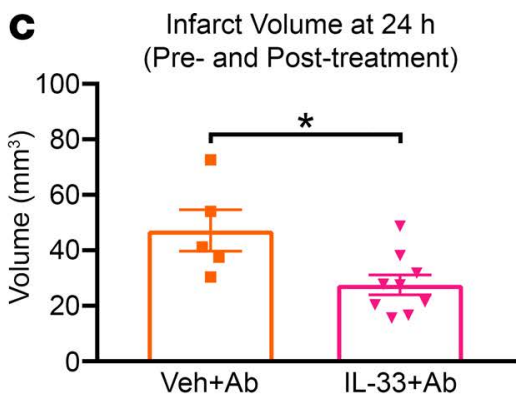

$\mathbf{F}$

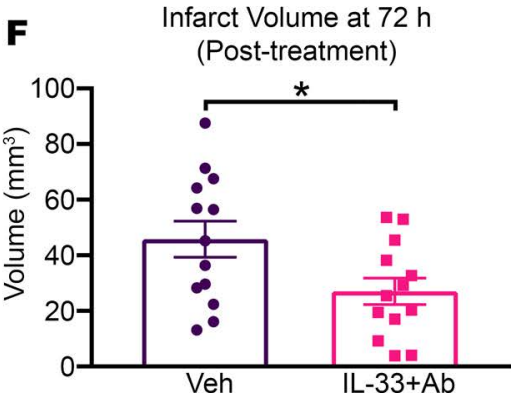

Figure 6. Infarct volumes in postischemic brains following combined treatment with antibiotics. Representative coronal brain sections from mice treated with a combination of (A) vehicle and antibiotics, (D) vehicle alone, or (B and E) IL-33 and antibiotics after stroke; and total infarct volumes at (C) 24 hours or (F) 72 hours after stroke (infarct areas are outlined in white). ${ }^{*} P<0.05$, Student's unpaired $t$ test. Infarct volume at 24 hours, $n=5-10$; infarct volume at 72 hours, $n=13-14$. Veh, vehicle; Ab, antibiotics.

at 3 days after stroke (Figure 7C), and the number of Tregs expressing IL-10 was 4-fold greater than in vehicle-treated mice (Figure 7D). IL-33 treatment also doubled the number of infiltrated Tregs expressing the IL-33 receptor ST2 as compared with control mice (Figure 7E).

Similar to the effect of high-dose IL-33 at 24 hours, low-dose IL-33 treatment had no effect on the total number of leukocytes (including $\mathrm{CD}^{+} \mathrm{T}$ cells) present in the brain at 3 days after stroke (our unpublished observations). Interestingly, low-dose IL-33 had no effect on the numbers of NK cells (Figure 7F) or NKT cells (our unpublished observations), but resulted in a 4-fold increase in IL-10-expressing NK cells in the brain at 72 hours (Figure $7 G$ ).

\section{Discussion}

There is growing evidence that a Th2-type inflammatory response can exert neuroprotective effects following cerebral ischemia via limiting inflammation-driven infarct progression. We recently found marked differences in the brain immune cell composition between Th1- and Th2-prone mouse strains following cerebral ischemia together with a milder stroke outcome in the latter strain (9). Here we have extended this finding by exploring the therapeutic potential of a recombinant Th2-type cytokine administered to provide neuroprotection in ischemic stroke.

There are several important findings of this study. First, we show that brief (daily for 2-3 days) systemic administration of the Th2-promoting cytokines IL-33 and IL-4 reduces acute brain injury after cerebral ischemia-reperfusion. Second, despite limiting infarct development, there was no effect on total immune cell infiltration, suggesting that IL-33 may have limited Th1-mediated injury by modulating the inflammatory environment, including the entry or activation state(s) of certain cell subsets. This included a reduced number of activated microglia; fewer monocytes, $3-\mathrm{NT}^{+} \mathrm{F} 4 / 80^{+}$proinflammatory microglia/macrophages, $\mathrm{CD}^{+} \mathrm{T}$ cells, $\mathrm{CD} 4^{+} \mathrm{NKT}$ cells, DN T cells, and NK cells; but more neutrophils and IL-10-expressing Tregs. Third, the DN T cells were increased after stroke in vehicle-treated mice, but not in IL-33-treated animals. We found that a subset of these DN T cells were present in much lower numbers than DN NKT cells, suggesting the IL-33-sensitive increase in DN T cells was instead mostly due to mucosa-associated invariant $\mathrm{T}$ cells and/or $\gamma \delta \mathrm{T}$ cells (32-34), which are also responsible for release of proinflammatory cytokines in disease settings $(35,36)$. Fourth, despite its neuroprotective effects, administration of IL-33 in the acute phase of stroke can also promote the acceleration of a 

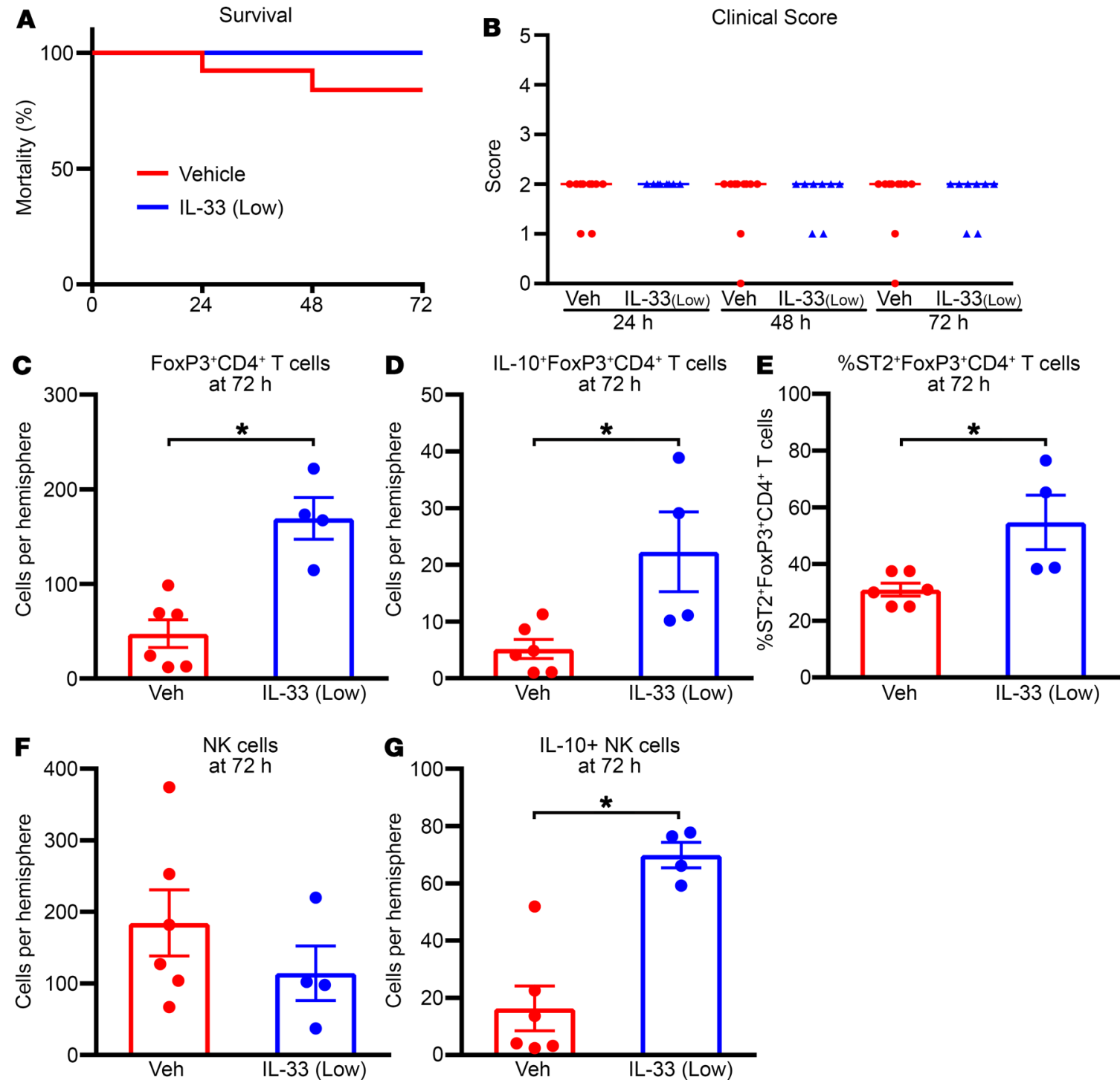

Figure 7. Effects of low-dose IL-33 on functional outcomes and leukocyte subsets at $\mathbf{7 2}$ hours. Survival (A) and clinical score (B) at 72 hours after stroke. Flow cytometric quantification of (C) FoxP3 ${ }^{+} \mathrm{CD} 4^{+} \mathrm{T}$ cells, (D) IL-10+FoxP+CD4+ $\mathrm{T}$ cells, (E) percentage of ST2-expressing FoxP3 ${ }^{+} \mathrm{CD} 4^{+} \mathrm{T}$ cells, (F) NK cells, and (C) IL-10+ NK cells in the ischemic hemisphere of IL-10 ${ }^{\text {eCFP } F o x p 3 ~}{ }^{\text {mRFP }}$ mice at 72 hours after stroke. Data are presented as mean \pm SEM. ${ }^{*} P<0.05$; Student's unpaired $t$ test. Flow cytometric analysis, $n=4-6$. Veh, vehicle.

systemic switch from a Th1- to a Th2-type inflammatory response that is associated with post-stroke immunosuppression, exacerbated bacterial infection in the lungs, and increased morbidity and mortality. Fifth, our detection of changes in immune cell numbers (due to stroke and/or IL-33) in the brain but not the blood at 24 hours suggests that the neuroprotection by IL-33 was related directly to its effects in the brain rather than any peripheral actions. Sixth, administration of antibiotics in combination with IL-33 prevents the increased post-stroke morbidity and mortality caused by the Th2-promoting cytokine while preserving its neuroprotective effects.

We investigated whether administering a novel cytokine, IL-33, can impact the outcome after ischemic stroke by modulating the post-stroke immune response in an antiinflammatory manner. Th2-promoting effects of IL-33 were confirmed by (i) increased expression of IL-5 and IL-13; (ii) attenuation of the stroke-induced increases in proinflammatory cytokines IL- $1 \beta$ and IL-6; and (iii) fewer $3-\mathrm{NT}^{+} \mathrm{F} 4 / 80^{+}$cells, consistent with fewer M1-polarized proinflammatory macrophages (37) in the post-stroke brain. Indeed, systemic administration of either IL-33 or the classical Th2 cytokine IL-4 markedly reduced the volume of cerebral infarction developing by 24 and 72 hours after ischemia-reperfusion. 
Tregs are established to modulate post-stroke inflammatory brain injury, possibly via an IL-10 signaling pathway (38). Our flow cytometric data demonstrate that IL-33 increased the numbers of Fox$\mathrm{p}^{+} \mathrm{CD}^{+}$Tregs, including IL-10-expressing Tregs in the brain, and that a higher percentage of these Tregs expressed the IL-33 receptor protein ST2 following IL-33 treatment. While a role of Tregs in mediating IL-33-induced neuroprotection has been questioned (39), other studies have asserted that Tregs are an important target of IL-33. For example, administration of IL-33 ameliorated experimental colitis by promoting a Foxp $3^{+}$Treg response, whereby the protective effect was abolished in the absence of Tregs (40). Furthermore, mice lacking ST2 exhibited Treg dysfunction consistent with an involvement of IL-33/ST2 signaling in Treg development and function (41). Further, IL-33 stimulated IL-2 secretion by dendritic cells to drive ST2 expression and expansion of $\mathrm{CD}^{+} \mathrm{Foxp}^{+}$Tregs (42). Indeed, ST2 ${ }^{+}$Tregs represent an important subset of activated Foxp $3^{+}$cells ( $\sim 10 \%$ of $\mathrm{CD} 3{ }^{+} \mathrm{CD} 4^{+} \mathrm{CD} 25^{+}$cells in thymus and spleen) (42). Finally, a recent study by Yang et al. demonstrated that neuroprotection by IL-33 in vitro is mediated by ST2-expressing microglia (43). Taken together, our data support the likely role of Tregs in IL-33-mediated neuroprotection after stroke.

We investigated another mechanism by which IL-33 could suppress the recruitment of lymphoid cells into the ischemic brain. The transmembrane chemokine CXCL16 and its receptor, CXCR6, are expressed in neurons, microglia, and astrocytes - cells that release soluble CXCL16 in response to proinflammatory stimuli (44). Soluble CXCL16 acts as a chemoattractant for activated CD8 ${ }^{+} \mathrm{T}$ cells, NKT cells, and Th1-polarized T cells that express CXCR6 $(45,46)$. We found immunohistochemical evidence for a marked increase in the expression of CXCL16 in the ischemic brain, which was attenuated by IL-33. These data may indicate that reduced CXCL16 expression contributed to the reduced chemoattraction by IL-33 of damaging lymphoid cells, such as $\mathrm{CD}^{+} \mathrm{T}$ cells and NKT cells, into the ischemic brain.

This study also addressed the importance of elevated Th2-related immune function on the severity of post-stroke infection. Stroke-associated pneumonia, secondary to the suppression of systemic immune cell function, is now understood to contribute to morbidity and mortality after ischemic stroke $(27,31)$. For example, stroke leads to leukopenia and splenic atrophy with fewer splenocytes, but more $\mathrm{CD}^{+} \mathrm{Foxp}^{+}$ Tregs not only in the spleen (47), but also in the ischemic hemisphere (2). Experimental and clinical data suggest that the degree of post-stroke immunosuppression is proportional to the severity of ischemic brain injury (48). Thus, we encountered adverse side effects of IL-33 administration after stroke manifested as increased 24-hour mortality, more severe bacterial infection of the lungs, and worsened functional impairment in survivors despite a $35 \%-50 \%$ smaller infarct volume than in vehicle-treated controls. We administered a dose of IL-33 ( $2 \mathrm{mg} / \mathrm{kg}$ per day for 2-3 days) that has been used safely for many weeks in other disease models (25). However, it seems likely that in our model of severe cerebral ischemia (60 minutes $\mathrm{MCAO}$ ), the adverse systemic effects of IL-33 were a consequence of its Th2-promoting immunosuppression to increase the susceptibility to bacterial infection, because these effects were abrogated by antibiotics. Subsequent studies to examine the role of IL-10 and/or Tregs over 72 hours avoided such effects by instead reducing the dose of IL-33 by $80 \%$ in combination with a milder stroke (45-minute MCAO).

In summary, we have demonstrated that short-term administration of a Th2-type cytokine, IL-33, exerts neuroprotection after cerebral ischemia, which involves an augmented Th2:Th1 cytokine profile, fewer proinflammatory and cytotoxic subsets of immune cells, and more IL-10-expressing Tregs infiltrating the injured brain. Further, our findings suggest that administration of such Th2-promoting therapies in severe cases of acute stroke is likely to be safer in combination with antibiotics to limit the risk of exacerbating post-stroke immunosuppression and bacterial infections.

\section{Methods}

Animals. This study fully adheres to the Animal Research: Reporting of In Vivo Experiments (ARRIVE) guidelines (49). All animal experiments were conducted in accordance with National Health and Medical Research Council of Australia guidelines for the care and use of animals in research and were approved by the Monash University Animal Ethics Committee. Mice had free access to water and food pellets before and after surgery.

A total of 456 male C57BL/6 mice provided by the Monash Animal Research Platform aged 8-12 weeks were studied. Mice were excluded from the study if they (i) died during the surgical procedure $(n=$ 18 ), (ii) experienced subarachnoid hemorrhage $(n=16)$, or (iii) were euthanized due to $<65 \%$ reduction in regional cerebral blood flow $(\mathrm{rCBF})$ during $\mathrm{MCAO}$ or $<50 \%$ recovery of rCBF within 10 minutes of 


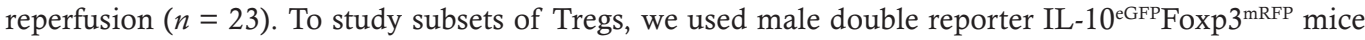
that were backcrossed on a C57BL/ 6 background for $>10$ generations and produced by our group (50), aged 12-16 weeks $(n=8)$. To study the role of Th1 immunity on the enhanced Th2 response subsequent to IL-33 therapy, we used male T-bet-deficient (T-bet ${ }^{-/-}$) mice backcrossed on a C57BL/6 background for $>10$ generations (obtained from Monash Animal Research Platform), aged 8-12 weeks, which lack the T-box transcription factor Tbx21 $(n=28)$; thus, $\mathrm{CD}^{+} \mathrm{T}$ cells from these mice do not produce the Th1type cytokine IFN but secrete elevated levels of Th2-type cytokines in response to inflammatory stimuli.

Treatments. Recombinant mouse IL-4 (5 $\mu \mathrm{g} ; 574306$, BioLegend) or IL-33 (2 $\mu \mathrm{g} ; 580508$, BioLegend) was injected intraperitoneally 24 hours before and immediately after reperfusion into C57BL/6 and T-bet ${ }^{-1}$ mice. Control mice were injected with the vehicle, $1 \%$ BSA. To determine the effect of preventing systemic bacterial infection, some animals received a combination of ampicillin (100 mg/kg) and gentamicin (4 $\mathrm{mg} / \mathrm{kg}$ ) subcutaneously. For experiments with a 24-hour end point, administration of antibiotics occurred 24 hours before, 1 hour after, and 8 hours after stroke. In experiments in which there was a 72-hour end point, animals received daily IL-33 injections starting immediately after reperfusion, and antibiotics were administered twice a day starting 1 hour after stroke. A separate cohort of animals (IL-10 ${ }^{\mathrm{eGFP}}$ Foxp3 ${ }^{\mathrm{mRFP}}$ mice) received $0.4 \mu \mathrm{g}$ IL-33 intraperitoneally 24 hours before and immediately after reperfusion. Mice were randomized into different treatment groups and experiments were conducted in a blinded fashion.

Transient focal cerebral ischemia. Focal cerebral ischemia was induced by transient intraluminal filament-induced occlusion of the right MCA, as described previously $(51,52)$. Mice were anesthetized with ketamine-xylazine ( 80 and $10 \mathrm{mg} / \mathrm{kg}$, respectively; intraperitoneally). Rectal temperature was monitored and maintained at $37.5^{\circ} \mathrm{C} \pm 0.5^{\circ} \mathrm{C}$ using an electronic temperature controller (Testronics) linked to a heat lamp throughout the procedure and until animals regained consciousness. Briefly, the right proximal common carotid artery was clamped, and a 6-0 nylon monofilament with silicone-coated tip (Doccol Corp.) was inserted and gently advanced into the distal internal carotid artery, 11-12 mm distal to the carotid bifurcation, occluding the MCA at the junction of the circle of Willis. Severe (typically $\sim 80 \%$ ) reduction in $\mathrm{rCBF}$ was confirmed using transcranial laser Doppler flowmetry (Perimed) in the area of cerebral cortex supplied by the MCA. The filament was then tied in place, and the clamp was removed. After 1 hour of cerebral ischemia, the monofilament was retracted to allow reperfusion for

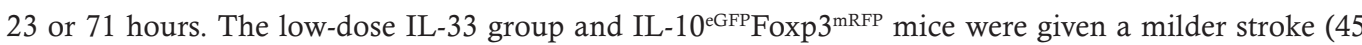
minutes of occlusion) to reduce acute mortality and enable 3 days of post-stroke survival. Reperfusion was confirmed by an immediate increase in $\mathrm{CCBF}$, which reached the preischemic level within 5 minutes. The wound was then closed, and the animal was allowed to recover. Regional CBF was recorded for 30 minutes of reperfusion. Sham-operated mice were anesthetized, and the right carotid bifurcation was exposed and dissected free from surrounding connective tissue, but no filament was inserted. All animals were administered $1 \mathrm{ml}$ sterile saline via a subcutaneous injection for rehydration after surgery. Gel nectar (Able Scientific) was placed inside the cage, and access to chow food and water was provided. All animals' boxes were placed on heat pads after surgery until euthanasia.

Clinical score assessment. At the end of the experiment (24 or 72 hours after induction of stroke/sham surgery), clinical assessment was performed by an observer blinded to experimental groups using a 6-point scoring system $(51,52)$ : 0 , normal motor function; 1 , flexion of torso and contralateral forelimb when mouse is lifted by the tail; 2 , circling when mouse held by the tail on a flat surface; 3 , leaning to one side at rest; 4, no spontaneous motor activity; 5 , death within the 23 - or 71-hour reperfusion period. A hanging wire test was also performed in which mice were suspended from a wire $30 \mathrm{~cm}$ high for up to 180 seconds, and the average time of 3 trials with 5-minute rest periods in between was recorded. Locomotor activity was assessed using the ANY-maze video tracking system, in which mice were placed in a $20 \times 20-\mathrm{cm}$ acrylic box with a floor made of steel rods spaced $8 \mathrm{~mm}$ apart and raised $10 \mathrm{~mm}$ above a base steel plate. Automated software detects and records exploratory parameters such as distance traveled during a 10-minute period.

Cerebral infarct and edema volumes. Mice were euthanized at 24 or 72 hours by isoflurane overdose, followed by decapitation. The brains were immediately removed and snap frozen with liquid nitrogen. Coronal sections $(30 \mu \mathrm{m})$ separated by $\sim 420 \mu \mathrm{m}$ were stained with thionine $(0.1 \%)$ to delineate the infarct. Images of the sections were captured with a CCD camera mounted above a light box. Infarct volume was quantified as described previously $(52,53)$ using image analysis software (NIH ImageJ) and corrected for brain edema, estimated using the following formula: corrected infarct volume $=[$ left hemisphere area $-($ right hemisphere 
area - right hemisphere infarct area) $\times$ (thickness of section + distance between sections)] $(54,55)$. Edema-corrected infarct volumes of individual brain sections were added to give a $3 \mathrm{D}$ approximation of the total infarct volume. Total, cortical, and subcortical infarct volumes were quantified individually.

Quantitative PCR. Mice were euthanized at 24 hours by isoflurane overdose and intracardially perfused with RNase-free PBS. The brain was removed from the skull, and after removal of the cerebellum and olfactory bulb, the right (ischemic) hemisphere was snap-frozen in liquid nitrogen. RNA was extracted using an RNeasy Mini Kit (QIAGEN) and reverse transcribed into cDNA using an $\mathrm{RT}^{2}$ First Strand Kit (QIAGEN). Levels of RNA expression of selected genes, including Il-1, $\mathrm{Il}-5$, Il-6, and Il-13, were measured using predesigned TaqMan gene expression assays. Gene expression data were normalized to the housekeeping gene GAPDH (Applied Bioscience) with the $\Delta \Delta \mathrm{Ct}$ method (56). Results were presented as fold change relative to vehicle-treated sham-operated mice.

Plasma cytokines. Mice were euthanized at 24 hours by isoflurane overdose, and blood was collected via cardiac puncture. Plasma samples were analyzed for the cytokines G-CSF, TNF, IL-6 and IL-5 using BD Cytometric Bead Array Cell Signaling (CBA) Flex Set System (BD Biosciences). Flex Set standard, capture bead master mix, and $\mathrm{PE}$ detection reagents were prepared as described in the Cell Signaling Master Buffer Kit (BD Biosciences). Samples were analyzed by FACSDiva Version 6.1.1 using forward scatter-A (FSC-A), FSC-W, side scatter-A(SSC-A), SSC-W, PE-A, APC-A, and APC-Cy7-A plots as described in the BD CBA Flex Set Templates for Flow Cytometers manual (BD Biosciences).

Flow cytometry. Mice were euthanized at 24 or 72 hours by isoflurane overdose, followed by blood collection by cardiac puncture, and the whole mouse was then intracardially perfused with PBS, and brain and spleen were collected. Leukocytes were purified from blood using red blood cell lysis buffer (155 mmol/1 $\mathrm{NH}_{4} \mathrm{Cl}, 10 \mathrm{mmol} / 1 \mathrm{KHCO}_{3}$, and $\left.3 \mathrm{mmol} / 1 \mathrm{EDTA}\right)$. Spleens were mechanically dissociated and passed through $70-\mu$ m nylon cell strainers (BD Falcon) to obtain a single-cell suspension. Cells were then lysed with red blood cell lysis buffer and washed with PBS containing 1\% BSA. The brain was removed from the skull and, after removal of the cerebellum and olfactory bulb, was separated into left (contralateral) and right (ischemic) hemispheres. Each hemisphere was dissociated mechanically in digestion buffer containing collagenase type XI $(125 \mathrm{U} / \mathrm{ml})$, hyaluronidase $(60 \mathrm{U} / \mathrm{ml})$, and collagenase type I-S (450 U/ml) in $\mathrm{Ca}^{2+} / \mathrm{Mg}^{2+}$-supplemented PBS (MilliporeSigma) and incubated at $37^{\circ} \mathrm{C}$ for 45 minutes with gentle agitation $(550 \mathrm{rpm})$. The mixture was then passed through $70-\mu \mathrm{m}$ nylon cell strainers to obtain a single-cell suspension. After washing with PBS $\left(350 \mathrm{~g}, 10\right.$ minutes at $\left.4^{\circ} \mathrm{C}\right)$, the cell pellet was resuspended in $3 \mathrm{ml} \mathrm{30 \%} \mathrm{Percoll} \mathrm{(GE} \mathrm{Healthcare),} \mathrm{underlaid} \mathrm{with} \mathrm{70 \%} \mathrm{Percoll,} \mathrm{and} \mathrm{centrifuged}$ for 20 minutes at $1,400 \mathrm{~g}$ at room temperature without the use of a brake. Cells at the interphase of 2 density gradients were collected and washed with PBS containing $1 \%$ BSA $\left(350 \mathrm{~g}, 10\right.$ minutes at $\left.4^{\circ} \mathrm{C}\right)$ for staining.

All cells were incubated with appropriate antibodies (listed in Tables 1 and 2 ) at $4^{\circ} \mathrm{C}$ in darkness for 20 minutes (Table 1) or 30 minutes (Table 2). After staining, cells were analyzed by LSRII flow cytometer (BD Biosciences; Table 1) or LSR Fortessa (BD Biosciences; Table 2), and FlowJo software (Tree Star Inc.). Countbright counting beads (Invitrogen) were included to define the absolute number of cells in the samples.

Gating strategy (Table 1): Forward and side scatters were used to identify single cells. Dead cells were excluded with Live/Dead markers (near infrared or aqua). Cells were gated for CD45 and $\mathrm{CD} 45^{\text {med }}$ populations as described previously (2). Microglial cells were identified as CD45 ${ }^{\text {med- }}$ $\mathrm{CD} 11 \mathrm{~b}^{+} \mathrm{F} 4 / 80^{+}$. $\mathrm{CD} 45^{\text {hi }}$ cell populations were then divided into myeloid and subdivided into monocytes $\left(C D 45^{\text {hi }} C D 11 b^{+} L y 6 C^{+}\right)$, macrophages $\left(C D 45^{\text {hi }} C D 11 b^{+} F 4 / 80^{+}\right)$, neutrophils $\left(C D 45^{\text {hi }} C D 11 b^{+}\right.$Ly$\left.6 \mathrm{C}^{+} \mathrm{Ly} 6 \mathrm{G}^{+}\right)$, and lymphoid cells, which included $\mathrm{T}$ cells $\left(\mathrm{CD} 45^{\mathrm{hi}} \mathrm{CD} 3^{+}\right), \mathrm{B}$ cells $\left(\mathrm{CD} 45^{\mathrm{hi}} \mathrm{CD} 19^{+}\right), \mathrm{NK}$

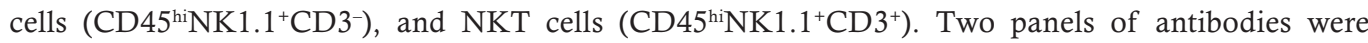
included for the study, one of which was employed with each animal. Panel 1 enabled the quantification of microglia and leukocytes with myeloid origin. This included macrophages $\left(\mathrm{F} 4 / 80^{+}\right)$, neutrophils $\left(\right.$ Ly $\left.6 \mathrm{G}^{+}\right)$, and monocytes $\left(\mathrm{Ly} 6 \mathrm{C}^{+}\right)$, which were further subdivided into Ly6 $\mathrm{C}^{\text {hi }}$ and $\mathrm{Ly} 6 \mathrm{C}^{\text {med }}$ monocytes Panel 2 enabled the identification and quantification of lymphoid cells consisting of B cells $\left(\mathrm{CD} 19^{+}\right)$, $\mathrm{NK}$ cells $\left(\mathrm{NK} 1.1^{+} \mathrm{CD} 3^{-}\right)$, NKT cells $\left(\mathrm{NK} 1.1^{+} \mathrm{CD}^{+}\right)$, and $\mathrm{T}$ cells $\left(\mathrm{CD}^{+}\right)$. T cells were further subdivided into $\mathrm{CD}^{+} \mathrm{T}$ cells, $\mathrm{CD} 8^{+} \mathrm{T}$ cells, $\mathrm{CD} 4^{+} \mathrm{CD} 8^{+} \mathrm{T}$ cells, and $\mathrm{CD} 4^{-} \mathrm{CD} 8^{-} \mathrm{DN} \mathrm{T}$ cells. $\mathrm{CD} 25^{+}$Tregs were also identified from $\mathrm{CD}^{+} \mathrm{T}$ cell populations. Fluorescence Minus One controls were also included in both panels to define positive populations for CD11b, Ly6C, F4/80, CD3, CD19, and CD25. 
Table 1. Summary of antibodies used for flow cytometry (C57BL/6)

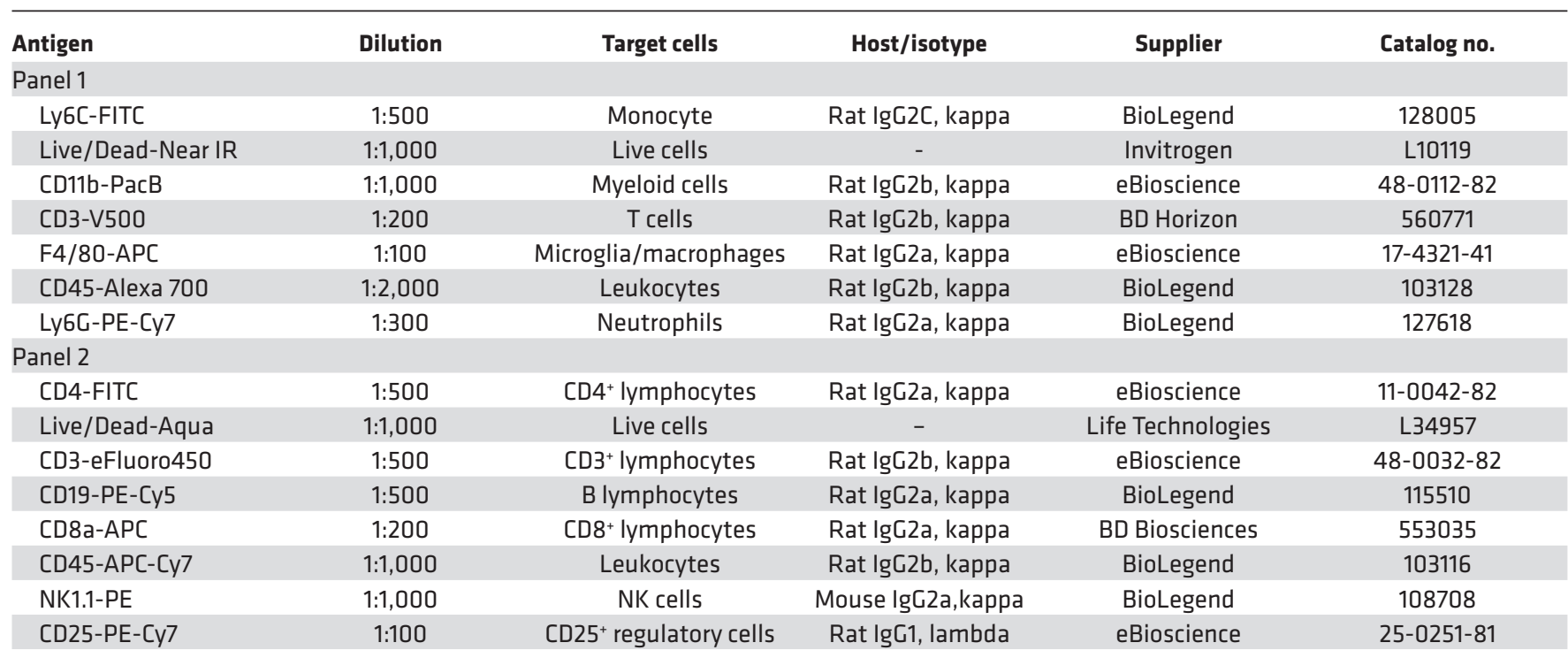

Gating strategy (Table 2): Forward and side scatters were used to identify single cells. Cells were gated for $\mathrm{CD} 45^{\mathrm{hi}}$ and then divided into $\mathrm{T}$ cells $\left(\mathrm{CD} 45^{\mathrm{hi}} \mathrm{CD} 3^{+}\right)$and $\mathrm{B}$ cells $\left(\mathrm{CD} 45^{\mathrm{hi}} \mathrm{B} 220^{+}\right) . \mathrm{CD} 45^{\mathrm{hi}} \mathrm{B} 220^{-}$cells were subdivided into $\mathrm{T}$ cells $\left(\mathrm{CD}^{+}\right)$, NK cells $\left(\mathrm{NK} 1.1^{+} \mathrm{CD}^{-}\right)$, and $\mathrm{NKT}$ cells $\left(\mathrm{NK} 1.1^{+} \mathrm{CD}^{+}\right)$. $\mathrm{T}$ cells were then further subdivided into $\mathrm{CD} 4^{+} \mathrm{T}$ cells and $\mathrm{CD}^{+} \mathrm{T}$ cells. $\mathrm{CD} 4^{+} \mathrm{T}$ cells were gated on IL-10 versus FoxP3 (quadrangle gates) and then on CD25 versus ST2 in all 4 populations. B cells, NK cells, and CD8 ${ }^{+} \mathrm{T}$ cells were also gated on IL-10 versus ST2 (quadrangle gates).

Immunofluorescence. Brain sections were immunofluorescently labeled for CXCL16 (membrane-bound CXCL16) or CD68 (activated microglia/macrophages [M/M cells]), or double-labeled for 3-NT (peroxynitrite marker) and F4/80 (M/M cell marker), to identify proinflammatory M/Ms (57). Frozen brains were sectioned $(10 \mu \mathrm{m})$ and thaw-mounted onto poly-L-lysine-coated slides. Multiple serial coronal brain sections that spanned the infarct region were taken for analysis. Sections were fixed in $4 \%$ paraformaldehyde for 15 minutes, washed in 0.01 M PBS ( $3 \times 10$ minutes), and blocked with $2 \%$ BSA (for CXCL16) or 10\% goat serum and a Mouse on Mouse Ig blocking reagent (Vector Laboratories) for 1 hour. Sections were then incubated with a polyclonal goat anti-CXCL16 antibody (1:800, AF503, R\&D Systems), a mouse-CD68 [ED1] antibody (1:100, ab31630, Abcam), or a mouse 3-NT antibody (1:50, ab61392, Abcam), and a rat anti-F4/80 antibody (1:100, 123147, BioLegend) overnight in a humidified chamber. The following day, sections were washed in $0.01 \mathrm{M}$ PBS $(3 \times 10$ minutes $)$ and

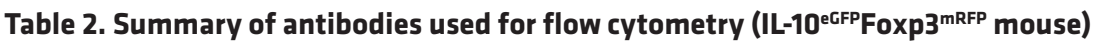

\begin{tabular}{|c|c|c|c|c|c|}
\hline CD25-APC & $1: 100$ & CD25+ regulatory cells & Rat IgG1, lambda & BioLegend & 102011 \\
\hline ST2-Biotin & $1: 100$ & IL-33 receptor & Rat IgC1 & MD Biosciences & 101001B \\
\hline CD4-BV510 & $1: 100$ & CD4+ Iymphocytes & Rat IgG2a, kappa & BioLegend & 100553 \\
\hline CD8a-PerCP & $1: 100$ & CD8+ Iymphocytes & Rat IgC2a, kappa & BioLegend & 100731 \\
\hline Streptavidin-BV421 & $1: 100$ & ST2-biotin antibody & & BioLegend & 405226 \\
\hline
\end{tabular}

Arm Ham, Armenian hamster. 
incubated in a chicken anti-goat Alexa Fluor 488 (1:200, A-21467, Invitrogen) secondary antibody or a goat anti-rat Alexa Fluor 594 (1:500, A-11007, Invitrogen) secondary antibody for 2 hours (3-NT- and F4/80-labeled sections only). Brain sections were then washed in $0.01 \mathrm{M}$ PBS $(3 \times 10$ minutes $)$ and incubated in biotinylated anti-mouse IgG reagent (Vector Laboratories) for 10 minutes. After washing in 0.01 M PBS $(3 \times 10$ minutes), fluorescein avidin DCS (Vector Laboratories) was applied onto the sections for 5 minutes. Sections were washed in 0.01 M PBS $(3 \times 10$ minutes $)$, coverslipped, and examined using an Olympus fluorescence microscope. Numbers of CXCL16 ${ }^{+}$cells, CD68 ${ }^{+}$cells, $3-\mathrm{NT}^{+}$cells, and $\mathrm{F} 4 / 80^{+}$cells in the right (ischemic) hemisphere were counted for each brain section by researchers blinded to treatment groups. For colocalization studies, data were presented as percentage of $3-\mathrm{NT}^{+}$ cells of total $\mathrm{F} 4 / 80^{+}$cells. All appropriate secondary antibody controls were performed to ensure that there was no nonspecific binding.

Bacteriological analysis. Animals were euthanized at 24 hours by inhalation of isoflurane and sprayed with $80 \%$ ethanol to maintain sterility. Blood was collected by cardiac puncture, followed by thoracotomy. The lungs, liver, and spleen were removed and homogenized in $1 \mathrm{ml}$ sterile PBS using an Omni Tissue Master 125 Homogenizer (Omni International). To determine CFU, $10 \mu 1$ tissue homogenate or blood was serially diluted and plated onto brain heart infusion (BHI) agar plates supplemented with $5 \%$ defibrinated horse blood (Australian Ethical Biologicals). Plates were incubated at $37^{\circ} \mathrm{C}$ for 18 hours, and bacterial colonies were counted.

Statistics. Values are presented as mean \pm SEM. Results of the hanging wire test, infarct volume, quantitative PCR (qPCR), plasma cytokine value, flow cytometry, immunohistochemistry, and bacteriological analysis were analyzed using 1-way ANOVA with Bonferroni's post hoc test with selected multiple comparisons or 2-tailed Student's unpaired $t$ test, as appropriate. The clinical score was expressed as the median result per group and was analyzed using a Kruskal-Wallis test with Dunn's post hoc test. A $P$ value less than 0.05 was considered statistically significant. Statistical analyses were carried out using GraphPad Prism (GraphPad Software).

Study approval. All animal studies and procedures were approved by the Monash University Animal Ethics Committee and fully complied with National Health and Medical Research Council of Australia guidelines for the care and use of animals in research.

\section{Author contributions}

SRZ, MG, CGS, and HAK designed the study. SRZ, MP, RS, CHYW, BRSB, SS, MG, HAK, HXC, and SL conducted various experiments, and acquired and analyzed data. AV, TM, SH, GRD, MAE, and TVA provided important reagents and valuable feedback on the manuscript. SRZ, CGS, and HAK wrote and edited the manuscript.

\section{Acknowledgments}

These studies were supported by Project Grants from the National Health and Medical Research Council of Australia (NHMRC) (APP1010984 and APP1064686). We also acknowledge support from NHMRC Senior Research Fellowships (GRD and CGS).

Address correspondence to: Christopher G. Sobey, Department of Physiology, Anatomy \& Microbiology, School of Life Sciences, HS2-335, La Trobe University, Kingsbury Drive, Bundoora, Victoria 3086, Australia. Phone: 61.3.9479.1316; Email: c.sobey@1atrobe.edu.au.

1. Gelderblom M, et al. Temporal and spatial dynamics of cerebral immune cell accumulation in stroke. Stroke. 2009;40(5):1849-1857.

2. Chu HX, et al. Immune cell infiltration in malignant middle cerebral artery infarction: comparison with transient cerebral ischemia. J Cereb Blood Flow Metab. 2014;34(3):450-459.

3. Stanimirovic D, Satoh K. Inflammatory mediators of cerebral endothelium: a role in ischemic brain inflammation. Brain Pathol. 2000;10(1):113-126

4. Vila N, Castillo J, Dávalos A, Chamorro A. Proinflammatory cytokines and early neurological worsening in ischemic stroke. Stroke. 2000;31(10):2325-2329.

5. Vila N, Castillo J, Dávalos A, Esteve A, Planas AM, Chamorro A. Levels of anti-inflammatory cytokines and neurological worsening in acute ischemic stroke. Stroke. 2003;34(3):671-675.

6. Engelbertsen D, et al. T-helper 2 immunity is associated with reduced risk of myocardial infarction and stroke. Arterioscler Thromb Vasc Biol. 2013;33(3):637-644. 
7. Paul WE, Zhu J. How are T(H)2-type immune responses initiated and amplified? Nat Rev Immunol. 2010;10(4):225-235.

8. Olson NC, Sallam R, Doyle MF, Tracy RP, Huber SA. T helper cell polarization in healthy people: implications for cardiovascular disease. J Cardiovasc Transl Res. 2013;6(5):772-786.

9. Kim HA, et al. Brain immune cell composition and functional outcome after cerebral ischemia: comparison of two mouse strains. Front Cell Neurosci. 2014;8:365.

10. Liu X, et al. Interleukin-4 is essential for microglia/macrophage M2 polarization and long-term recovery after cerebral ischemia. Stroke. 2016;47(2):498-504.

11. Zhao X, Wang H, Sun G, Zhang J, Edwards NJ, Aronowski J. Neuronal interleukin-4 as a modulator of microglial pathways and ischemic brain damage. J Neurosci. 2015;35(32):11281-11291.

12. de Bilbao F, et al. In vivo over-expression of interleukin-10 increases resistance to focal brain ischemia in mice. J Neurochem. 2009;110(1):12-22.

13. Ooboshi $\mathrm{H}$, et al. Postischemic gene transfer of interleukin-10 protects against both focal and global brain ischemia. Circulation. 2005;111(7):913-919.

14. Pérez-de Puig I, et al. IL-10 deficiency exacerbates the brain inflammatory response to permanent ischemia without preventing resolution of the lesion. J Cereb Blood Flow Metab. 2013;33(12):1955-1966.

15. Schmitz J, et al. IL-33, an interleukin-1-like cytokine that signals via the IL-1 receptor-related protein ST2 and induces T helper type 2-associated cytokines. Immunity. 2005;23(5):479-490.

16. Coyle AJ, et al. Crucial role of the interleukin 1 receptor family member T1/ST2 in T helper cell type 2-mediated lung mucosal immune responses. J Exp Med. 1999;190(7):895-902.

17. Smithgall MD, Comeau MR, Yoon BR, Kaufman D, Armitage R, Smith DE. IL-33 amplifies both Th1- and Th2-type responses through its activity on human basophils, allergen-reactive Th2 cells, iNKT and NK cells. Int Immunol. 2008;20(8):1019-1030.

18. Zhao W, Hu Z. The enigmatic processing and secretion of interleukin-33. Cell Mol Immunol. 2010;7(4):260-262.

19. Seki K, et al. Interleukin-33 prevents apoptosis and improves survival after experimental myocardial infarction through ST2 signaling. Circ Heart Fail. 2009;2(6):684-691.

20. Miller AM, et al. IL-33 reduces the development of atherosclerosis. J Exp Med. 2008;205(2):339-346.

21. Seo DH, et al. Interleukin-33 regulates intestinal inflammation by modulating macrophages in inflammatory bowel disease. $S c i$ Rep. 2017;7(1):851.

22. Fu AK, et al. IL-33 ameliorates Alzheimer's disease-like pathology and cognitive decline. Proc Natl Acad Sci USA. 2016;113(19):E2705-E2713.

23. Jiang HR, et al. IL-33 attenuates EAE by suppressing IL-17 and IFN- $\gamma$ production and inducing alternatively activated macrophages. Eur J Immunol. 2012;42(7):1804-1814.

24. Milovanovic M, et al. IL-33/ST2 axis in inflammation and immunopathology. Immunol Res. 2012;52(1-2):89-99.

25. Komai-Koma M, Gilchrist DS, McKenzie AN, Goodyear CS, Xu D, Liew FY. IL-33 activates B1 cells and exacerbates contact sensitivity. J Immunol. 2011;186(4):2584-2591.

26. Abrahamsohn IA, da Silva AP, Coffman RL. Effects of interleukin-4 deprivation and treatment on resistance to Trypanosoma cruzi. Infect Immun. 2000;68(4):1975-1979.

27. Brait VH, Arumugam TV, Drummond GR, Sobey CG. Importance of T lymphocytes in brain injury, immunodeficiency, and recovery after cerebral ischemia. J Cereb Blood Flow Metab. 2012;32(4):598-611.

28. Gu L, Xiong X, Zhang H, Xu B, Steinberg GK, Zhao H. Distinctive effects of T cell subsets in neuronal injury induced by cocultured splenocytes in vitro and by in vivo stroke in mice. Stroke. 2012;43(7):1941-1946.

29. Wong $\mathrm{CH}$, et al. Prolonged activation of invariant natural killer T cells and TH2-skewed immunity in stroke patients. Front Neurol. 2017;8:6.

30. Theodorou GL, et al. T helper 1 (Th1)/Th2 cytokine expression shift of peripheral blood CD4+ and CD8+ T cells in patients at the post-acute phase of stroke. Clin Exp Immunol. 2008;152(3):456-463.

31. Koennecke HC, et al. Factors influencing in-hospital mortality and morbidity in patients treated on a stroke unit. Neurology. 2011;77(10):965-972.

32. D'Acquisto F, Crompton T. CD3+CD4-CD8- (double negative) T cells: saviours or villains of the immune response? Biochem Pharmacol. 2011;82(4):333-340.

33. Reantragoon R, et al. Antigen-loaded MR1 tetramers define T cell receptor heterogeneity in mucosal-associated invariant T cells. J Exp Med. 2013;210(11):2305-2320.

34. Martin E, et al. Stepwise development of MAIT cells in mouse and human. PLoS Biol. 2009;7(3):e54.

35. Zlotnik A, Godfrey DI, Fischer M, Suda T. Cytokine production by mature and immature CD4-CD8- T cells. Alpha beta-T cell receptor+ CD4-CD8- T cells produce IL-4. J Immunol. 1992;149(4):1211-1215.

36. Crispín JC, et al. Expanded double negative T cells in patients with systemic lupus erythematosus produce IL-17 and infiltrate the kidneys. J Immunol. 2008;181(12):8761-8766.

37. Hu X, et al. Microglia/macrophage polarization dynamics reveal novel mechanism of injury expansion after focal cerebral ischemia. Stroke. 2012;43(11):3063-3070.

38. Liesz A, et al. Regulatory T cells are key cerebroprotective immunomodulators in acute experimental stroke. Nat Med. 2009;15(2):192-199.

39. Korhonen P, et al. Immunomodulation by interleukin-33 is protective in stroke through modulation of inflammation. Brain Behav Immun. 2015;49:322-336.

40. Duan L, et al. Interleukin-33 ameliorates experimental colitis through promoting Th2/Foxp3 ${ }^{+}$regulatory T-cell responses in mice. Mol Med. 2012;18:753-761.

41. Zdravkovic N, Shahin A, Arsenijevic N, Lukic ML, Mensah-Brown EP. Regulatory T cells and ST2 signaling control diabetes induction with multiple low doses of streptozotocin. Mol Immunol. 2009;47(1):28-36.

42. Matta BM, et al. IL-33 is an unconventional Alarmin that stimulates IL-2 secretion by dendritic cells to selectively expand IL-33R/ST2+ regulatory T cells. J Immunol. 2014;193(8):4010-4020.

43. Yang Y, et al. ST2/IL-33-Dependent Microglial Response Limits Acute Ischemic Brain Injury. J Neurosci. 
2017;37(18):4692-4704.

44. Ludwig A, et al. Enhanced expression and shedding of the transmembrane chemokine CXCL16 by reactive astrocytes and glioma cells. J Neurochem. 2005;93(5):1293-1303

45. Matloubian M, David A, Engel S, Ryan JE, Cyster JG. A transmembrane CXC chemokine is a ligand for HIV-coreceptor Bonzo. Nat Immunol. 2000;1(4):298-304.

46. Wilbanks A, et al. Expression cloning of the STRL33/BONZO/TYMSTRligand reveals elements of CC, CXC, and CX3C chemokines. J Immunol. 2001;166(8):5145-5154.

47. Offner H, et al. Splenic atrophy in experimental stroke is accompanied by increased regulatory T cells and circulating macrophages. J Immunol. 2006;176(11):6523-6531.

48. Hug A, et al. Infarct volume is a major determiner of post-stroke immune cell function and susceptibility to infection. Stroke. 2009;40(10):3226-3232.

49. Kilkenny C, Browne W, Cuthill IC, Emerson M, Altman DG, National Centre for the Replacement, Refinement Reduction of Amimals in Research. Animal research: reporting in vivo experiments - the ARRIVE guidelines. J Cereb Blood Flow Metab. 2011;31(4):991-993

50. Brockmann L, et al. IL-10 receptor signaling is essential for TR1 cell function in vivo. J Immunol. 2017;198(3):1130-1141.

51. Brait $\mathrm{VH}$, et al. Mechanisms contributing to cerebral infarct size after stroke: gender, reperfusion, $\mathrm{T}$ lymphocytes, and Nox2-derived superoxide. J Cereb Blood Flow Metab. 2010;30(7):1306-1317.

52. Jackman KA, Miller AA, De Silva TM, Crack PJ, Drummond GR, Sobey CG. Reduction of cerebral infarct volume by apocynin requires pretreatment and is absent in Nox2-deficient mice. Br J Pharmacol. 2009;156(4):680-688.

53. Kim HA, et al. Brain infarct volume after permanent focal ischemia is not dependent on Nox2 expression. Brain Res 2012;1483:105-111.

54. Tsuchiya D, Hong S, Kayama T, Panter SS, Weinstein PR. Effect of suture size and carotid clip application upon blood flow and infarct volume after permanent and temporary middle cerebral artery occlusion in mice. Brain Res. 2003;970(1-2):131-139.

55. Xia CF, et al. Postischemic brain injury is exacerbated in mice lacking the kinin B2 receptor. Hypertension. 2006;47(4):752-761

56. Schmittgen TD, Livak KJ. Analyzing real-time PCR data by the comparative C(T) method. Nat Protoc. 2008;3(6):1101-1108

57. Chu HX, Broughton BR, Kim HA, Lee S, Drummond GR, Sobey CG. Evidence that Ly6C(hi) monocytes are protective in acute ischemic stroke by promoting M2 macrophage polarization. Stroke. 2015;46(7):1929-1937. 